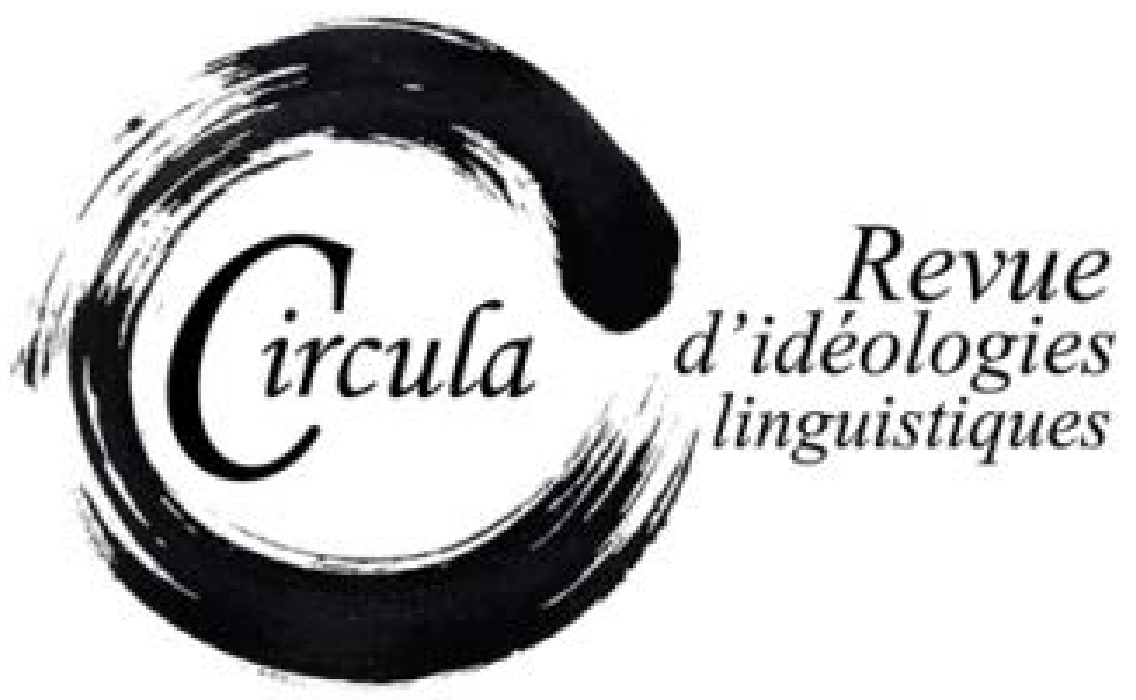

TITRE: UN CASO DI CRONACA LINGUISTICA PER LA SCUOLA. IDA BACCINI E LE ORIGINI DI LINGUA ITALIANA Auteur(s): Monastra, Alessandra, UniversitÀ degli STUdI DI Messina Revue: Circula, NumÉRO 5

PAGES: $43-66$

ISSN: 2369-6761

Directeurs: Wim Remysen, SABINe SCHWARZE ET JuAN ANTONIO ENNIS

URI: HTTP://HDL.HANDLE.NET/11143/11228

DOI: HTTPS://DOI.ORG/10.17118/11143/11228 


\title{
Un caso di cronaca linguistica per la scuola. Ida Baccini e le origini di Lingua Italiana ${ }^{1}$
}

\author{
Alessandra Monastra, Università degli studi di Messina \\ alessandramonastra@me.com
}

\begin{abstract}
Riassunto: L'articolo indaga le origini di Lingua italiana: cronaca linguistica a carattere preminentemente didattico, pubblicata nel supplemento della rivista La Rassegna Scolastica dal 1896 al 1908. La ricerca si suddivide in tre sezioni: la prima osserva il panorama pubblicistico scolastico italiano del secondo Ottocento; la seconda analizza la rivista, il supplemento e i caratteri principali della rubrica Lingua italiana; la terza, più ampia, presenta la figura di Ida Baccini, prima compilatrice della cronaca, nonché insegnante e scrittrice. Le riflessioni normative esposte dalla Baccini in rubrica sono state, inoltre, confrontate (tramite apposite tabelle sinottiche) con quelle presenti in alcune grammatiche postunitarie, adottate negli istituti scolastici dell'epoca. L'intento principale è quello di delineare il profilo ideologico-linguistico della Baccini, che si presenta, in definitiva, di matrice principalmente manzoniana, con sporadici tratti filotradizionalisti.
\end{abstract}

Parole chiave: didattica della lingua italiana; cronaca linguistica; Ida Baccini; norma; grammatica; istruzione scolastica

\begin{abstract}
This article investigates the origins of Lingua Italiana, a primarily didactic linguistic chronicle, published as an addendum in La Rassegna Scolastica magazine from 1896 to 1908. The research is subvided into three sections: the first section surveys the Italian journalistic and school scene of the second half of the eighteenth century; the second section analyzes the magazine, the addendum and the main characters of Lingua Italiana; the third and largest one introduces the figure of Ida Baccini, first compiler of the chronicle as well as teacher and writer. The normative reflections presented by the compiler in the column were also compared to those present in some post-unification grammars, used in the educational institutions of the period. The main aim is outlining the ideological-linguistic profile of Ida Baccini that ultimately reveals the Manzonian influence with some sporadic traits of pro-traditionalist thinking.
\end{abstract}

Keywords: teaching Italian language; linguistic chronicle; Ida Baccini; standard; grammar; education

1. Desidero ringraziare vivamente il prof. Fabio Rossi per avermi seguito nella stesura di questo lavoro e per averlo arricchito con i suoi preziosi suggerimenti. Ringrazio inoltre la prof.ssa Elisa De Roberto per avermi permesso di fruire del suo lavoro quando era ancora in stampa e per avermi consigliato in merito al reperimento delle opere di Ida Baccini. 


\section{Introduzione. I periodici scolastici italiani del secondo Ottocento}

L'importanza dell'editoria scolastica nel secondo Ottocento, dettagliatamente documentata da Giorgio Chiosso (1992), è ben ravvisabile nelle parole con cui Giovanni Parato, direttore del periodico scolastico torinese La guida del maestro elementare italiano, si rivolgeva ai suoi lettori nell'ottobre del 1867:

[...] niuno che sia di giudizio equo e disinteressato può contestare la utilità dei giornali pratici d'istruzione, purché siano ben condotti. Giornali pratici grandemente diffusi ha l'Inghilterra, la Germania e più ancora la Francia. E noi crediamo che maggior bisogno di tutti ne abbia l'Italia, dove l'edificio dell'istruzione primaria e popolare non fu certo la lenta opera di anni, ma tutto si è fatto, e dovuto fare in pochissimo tempo. (Parato, 1867: 801-802)

Un simile discorso era dettato dalla lucida consapevolezza che il sistema scolastico presente in paesi quali Francia, Germania o Inghilterra fosse di gran lunga più funzionale rispetto a quello italiano: in Italia, infatti, si contavano all'incirca 17 milioni di analfabeti su un totale di 23 milioni di abitanti. In un contesto siffatto, ben si comprende come la scuola non potesse far altro che riflettere l'estrema disomogeneità del neonato Stato italiano.

È dunque evidente l'importanza di una pubblicistica specializzata (ovvero di riviste specializzate) che veicolasse informazioni ministeriali, programmi scolastici e sezioni interamente dedicate alla didattica pratica, con l'immediato intento di colmare le lacune dei maestri e, insieme, di uniformare, anche se in maniera piuttosto sommaria, i diversi cicli scolastici e i relativi metodi d'insegnamento.

Nonostante il generoso impegno editoriale in questa direzione, dei 35 periodici scolastici pubblicati tra il 1860 e il 1866 (molti dei quali con finalità esclusivamente didattica), soltanto meno della metà riuscirono a sopravvivere o, comunque, ad avere una circolazione non municipalistica ma di più ampio respiro. Promotrici di tale diffusione furono città come Milano, Napoli e Torino: basterà ricordare L'educatore italiano (1861-1885) per il capoluogo lombardo; L'amico delle scuole popolari (1861-1876) per quello campano e La guida del maestro elementare italiano (1864-1897), insieme a L'osservatore scolastico (1865-1899), per l'allora capitale subalpina. Quest'ultima, già nel 1853, aveva visto la nascita de L'istitutore, periodico di capitale importanza, fondato da Domenico Berti e diretto da Giovanni Lanza, che rappresentava ancora una voce molto influente nel panorama scolastico. Torino rimase il polo principale per la diffusione della pubblicistica scolastica ancora per tutto il decennio successivo: gli anni '70, infatti, la videro primeggiare in maniera assoluta su città quali Milano, Palermo, Firenze, Genova e Roma; quest'ultima, addirittura, si collocava in ultima posizione con un solo periodico (Ottino, 1875). Tale primato proseguiva una tradizione che, viva fin dagli anni '40, fu mantenuta fino al 1890 circa, periodo in cui l'egemonia torinese cedette il passo alla stampa lombarda e romana. Durante gli anni ' 80 anche la città di Firenze svolse un ruolo di primaria importanza 
in questo settore; ruolo dovuto soprattutto alla presenza sia di importanti istituzioni educative e culturali, sia di una folta schiera di validi funzionari².

Fu proprio in questo decennio di profondi cambiamenti non solo culturali, ma anche pedagogici, che i periodici scolastici subirono un sostanziale rinnovamento, manifestatosi principalmente con il superamento del fine quasi esclusivamente didattico; si legga, a tal proposito, ciò che nel 1880 il Liberto scriveva su La scuola italiana di Torino (1880-1881):

[...] Oggi, fortunatamente, questo giornale non basta più. Il maestro vuole ben altro: ha coscienza di sé, ha ragione di offendersi che altri lo consideri come una macchinetta che ripeterà macchinalmente quanto il giornale scolastico verrà a sciorinargli dinanzi ogni sabato. Si vuole quella stampa che sa elevarsi, sviscerare le grandi questioni, preparare al maestro la via, ma preparargliela in modo che studii indefessamente, che si liberi dalle pastoie, che lasci le grucce e cammini diritto alla meta. (Liberto, 1880-1881: 86-87)

La svolta è testimoniata da periodici quali L'unione dei maestri elementari d'Italia di Torino (18701917), La rivista dell'istruzione primaria, popolare e professionale (1880-1881) e La luce (1877-1881) di Roma, o anche l'Archivio di pedagogia e di scienze affini (1876-1883) di Palermo.

Gli anni '80 videro contrapporsi Stato e Chiesa anche per questioni legate all'ambito scolastico: molti ambienti aspiravano ad una laicizzazione del sapere, che sarebbe servita a rendere più maturi gli animi degli italiani, chiamati ad abbracciare gli ideali liberali e borghesi, allora in costante diffusione. La pubblica amministrazione si rese ben presto conto che un tale risultato poteva essere raggiunto esclusivamente attraverso l'opera di maestri e di professori, i quali dovevano essere, quindi, sensibilizzati repentinamente: furono così varati, tra il 1875 e il 1885, diversi provvedimenti volti a migliorare il loro tenore di vita e a riconoscere il loro ruolo fondamentale. La scuola venne considerata, per dirla col Barbagli, «agente di socializzazione politica» (Barbagli, 1974: 87); veicolo di una pedagogia laica, progressista e sollecitata dal potere politico. In un contesto simile, non è da escludere che la pubblicistica scolastica abbia risentito non soltanto dei venti di rinnovamento, ma anche di veri e propri tentativi di condizionamento, dettati dall'amministrazione centrale e periferica: è ben nota, infatti, la prassi, perpetrata dalle diverse fazioni politiche che si avvicendavano al potere, di impadronirsi di testate importanti con lo scopo di pilotare l'opinione pubblica a proprio vantaggio.

2. Sull'ambiente culturale e pedagogico fiorentino ed i suoi rapporti con l'editoria scolastica si rinvia a Porciani (1893). 
Un certo potenziamento si registrò anche nel campo dell'istruzione secondaria, nel cui ambito, accanto a pubblicazioni di carattere generale, fiorirono anche periodici specializzati nell'insegnamento di discipline quali la matematica, la ginnastica e le lingue straniere: tra queste ultime si ricorderà quantomeno La lingua francese nelle scuole secondarie d'Italia. Pubblicata a Torino dal 1883 al 1904, essa dava ampio spazio alla didattica pratica (corredata anche da esercizi), ma si proponeva, nel contempo, come un efficacissimo mezzo per indagare la società e la cultura francese.

Gli anni '90 ebbero inizio con la fondazione dell'Associazione della stampa scolastica nazionale, unitasi immediatamente al Circolo dei giornalisti italiani: tale connubio mostrava esplicitamente la ferma volontà di proseguire sulla via di un giornalismo scolastico moderno, nel cui ambito si producevano riviste sostenute dagli editori, assai più complete ed organiche, dove, accanto alla consueta sezione didattica, non potevano mancare le informazioni letterarie e le notizie politiche. Ispirata a quest'aria di rinnovamento fu la testata I diritti della scuola (1899), rivista che inglobava al suo interno molteplici contenuti e che si proponeva, dunque, come uno strumento in grado di soddisfare esigenze ed interessi diversificati.

\section{I maggiori centri di produzione negli anni 1880 -1890}

Roma iniziò a manifestare un'importante vitalità pubblicistica solo dopo il 1880: fu allora che si realizzò una ricca fioritura di riviste e giornali che nel giro di cinque anni le avrebbe permesso di divenire la prima città italiana per numero di testate stampate.

Tra queste basterà menzionare L'avvenire dei maestri elementari italiani (1880-1897); /l nuovo educatore (1881-1902); /l corriere scolastico (1890-1902); Il rinnovamento scolastico (1892-1899) e La scuola educatrice (1894-1910). Con la sola eccezione di quest'ultima, diretta espressione dell'ambiente cattolico romano, si trattava di pubblicazioni il cui scopo cardine era la promozione di una scuola laica, sostenuta con maggior vigore dallo Stato. Vi si proponevano iniziative miranti a raccogliere l'eredità dei periodici torinesi e fiorentini, i quali avevano manifestato, nel recente passato, un forte interesse nei confronti dell'organizzazione scolastica a livello nazionale, non fermandosi esclusivamente al ristretto ambito locale.

Stessa sorte toccò al capoluogo lombardo, nel quale si registrò un sensibile incremento delle testate prodotte: a titoli già molto conosciuti e rinomati come L'educatore italiano (1861-1885) e Patria e famiglia (1875), si aggiunse /l risveglio educativo (1884-1901), punto di riferimento degli ambienti pedagogici sensibili al positivismo. Attorno a quest'ultimo titolo si moltiplicarono delle vere e proprie catene di periodici, dovute, in massima parte, anche al costante perfezionamento delle tecniche di stampa, le quali, riducendo notevolmente i costi, permisero una circolazione di più ampio raggio. 
Anche il Meridione presentava un'interessante vitalità: prima Napoli, poi Palermo costituirono i poli centrali per la stampa scolastica; tuttavia, sarebbe forse più corretto parlare di un fervente dinamismo pubblicistico manifestatosi nell'intera Sicilia, la quale, con 65 testate, andava ad occupare il terzo posto in graduatoria tra le regioni italiane, dietro Lombardia e Piemonte. Tale situazione, descritta dal Chiosso come «un singolare ed interessante caso di policentrismo editoriale» (Chiosso, 1992: 37), fu dovuta, in massima parte, ad una sorprendente spinta fornita da ambienti didattici particolarmente attivi nell'isola3. Oltre al già citato Archivio di pedagogia e di scienze affini (1876-1883), la cui eredità fu recepita dalla Cronaca dell'istruzione pubblica e privata (1884-1885) e da L'avvenire educativo (1886-1898), sarà opportuno menzionare anche Scuola e famiglia, periodico sorto nel 1873 e destinato a prosperare fino al 1906.

\section{Firenze: La Rassegna Scolastica}

Edita dalla Bemporad ${ }^{4}$ e diretta da Domenico Giannitrapani ${ }^{5}$, La Rassegna Scolastica (1895-1908) rappresentò un importante esempio di rivista moderna. Ricca di contenuti eterogenei, ben articolata e a diffusione nazionale, si distinse nel panorama fiorentino degli anni '90 in quanto unica testata attenta agli importanti cambiamenti in atto nella scuola. Sul fronte pedagogico-didattico si allineò a posizioni positivistiche, su quello politico si affiancò, invece, ad un moderato liberalismo; così si legge nella dichiarazione d'intenti sul primo fascicolo (I, n $1,1^{\circ}$ ottobre 1895$)$, nella quale si esprimeva il proposito di fondare:

3. Sulla vita scolastica dell'isola in quegli anni cf. Bonetta (1981; 1990: 231-42).

4. Un timido ingresso della casa editrice nel mondo della pubblicistica scolastica si registrò nel 1891 con la rivista // Caffè, quindicinale didattico a diffusione locale. Di breve durata (solo pochi mesi) e povero di contenuti, fu principalmente destinato a studenti e professori delle scuole secondarie classiche. Il periodico era di proprietà del direttore Marco Visciola; a ricondurlo alla Bemporad fu solo il marchio impresso sul frontespizio del primo fascicolo. La casa editrice si legò stabilmente al mondo dei periodici pedagogici prima con La Rassegna Scolastica (1895-1908), poi con II Rinnovamento scolastico (1923-1925) e infine con / diritti della scuola, di cui acquistò nel 1926 una quota che mantenne fino al 1935.

5. Domenico Giannitrapani nacque a Trapani nel 1836, si laureò in Ingegneria presso l'Università di Palermo e nel 1860 si unì ai garibaldini sbarcati a Marsala; in seguito entrò tra le fila dell'esercito italiano. Non stupisce, quindi, che il suo esordio come scrittore sia legato ad un testo di argomento militare (Lo stato maggiore del genio nell'Esercito italiano, estr. dalla Rivista militare italiana, Roma 1879). Trasferitosi a Bologna, iniziò a coltivare interessi geografici che si manifestarono in diversi testi, tra i quali Geografia della provincia di Bologna, (1883) e Manuale di geografia ad uso delle scuole secondarie (1886-1887). Per la sua predisposizione alla didattica della geografia fu fondamentale l'incontro con l'editore E. Bemporad, il quale, a partire dal 1889, aveva rilanciato le pubblicazioni della «Biblioteca scolastica». Appartengono a tale collezione le sue opere più fortunate: Geografia per le scuole secondarie (1895); Geografia per le scuole ginnasiali, tecniche e normali (1897); Nozioni di geografia per le scuole elementari (1890-1891) e Geografia dell'Europa (1899). Il trasferimento a Firenze, nel 1892, gli permise inoltre di affiancarsi alla nuova generazione di geografi italiani, sorta attorno all'eminente figura di Giovanni Marinelli; fu in questo periodo che il G. si interessò alla geografia umana: risalgono ad allora i saggi Il monte Erice, oggi S. Giuliano: paesaggio, storia e costumi (1892) e La colonizzazione della Patagonia (in Rassegna nazionale, 16 nov. 1899, p. 266-272). Collaborò, fin dal primo fascicolo, alla Rivista geografica italiana e ricoprì il ruolo di consigliere nella Società di studi geografici e coloniali. 
[...] un periodico scolastico che non servisse alcuna chiesuola, ma fosse schiettamente italiano e indipendente e intendesse soprattutto al progresso degli studi seguendo i veri bisogni dell'istruzione e dell'educazione, per modo di innalzare a dignità di scienza quanto concerne la scuola, rendendola essenzialmente nazionale e animata dallo spirito moderno, senza rinunziare alle sue antiche tradizioni. (Chiosso, 1992: 343)

Inizialmente, il periodico fu genericamente rivolto alla classe degli insegnanti, ma in seguito destinò esplicitamente le proprie pagine ai maestri elementari, per i quali, a partire dal 1896, aggiunse anche un supplemento didattico, del quale si dirà più avanti.

Nelle pagine della Rassegna si susseguirono articoli riguardanti l'insegnamento scolastico elementare e secondario, la pedagogia e la politica scolastica, non mascherando mai la chiara consapevolezza dell'inadeguatezza della legge Casati, alla quale, tuttavia, non fu mai contrapposta un'univoca proposta di riforma. Altri importanti temi trattati furono: l'obbligo scolastico; la questione pensionistica del personale docente; l'avocazione della scuola elementare allo Stato e i programmi scolastici elementari. Si trattava, dunque, di una rivista principalmente, o meglio quasi esclusivamente, interessata alla questione magistrale: fatto, questo, che risultava essere di capitale importanza in un'epoca che non riconosceva il valore professionale e sociale dei maestri, che versavano in gravi condizioni di miseria culturale ed economica. Una folta schiera di collaboratori contribui a rendere la Rassegna una delle più autorevoli e diffuse riviste didattiche del tempo; tra essi Ruggero Bonghi, Ferdinando Martini, Ida Baccini, Pietro Dazzi, Ermenegildo Pistelli.

L'impegno profuso da Domenico Giannitrapani, direttore del periodico, nonché persona di grande spessore culturale e scientifico, risultò indispensabile al punto che la rivista non riuscì a sopravvivere che un solo anno al suo abbandono. Attraverso la Rassegna egli mostrò apertamente, oltre alla manifesta passione per gli studi geografici, il suo spirito patriottico e l'intento di diffondere l'istruzione tra il popolo, ponendosi sempre a fianco della classe docente, al di fuori di qualsiasi logica partitica:

[...] l'istruzione è un patrimonio sacro alla nazione ed alla civiltà e deve essere tenuta al di sopra e all'infuori di ogni spirito partigiano [...] gli educatori non formano un partito, ma sono i rappresentanti della parte più sana ed eletta della nazione, intemerati e saldi come torre che non crolla. (Giannitrapani, 1898-1899: 478)

Consapevole della necessità di creare un fronte comune d'insegnanti, a partire dal 1887 schierò apertamente il giornale a favore dei diritti della categoria: si trattò di un percorso intrapreso contemporaneamente da diverse testate e che diede un forte impulso alla nascita, nel 1901, dell'Unione Magistrale Nazionale (UNM). Coerentemente con l'interesse mostrato in tale direzione, la rivista seguì da vicino la crescita del nuovo organo, destinando una rubrica specifica alle attività dell'UNM. 
Altro ambizioso progetto perseguito con grande dedizione ed interesse dal Giannitrapani fu quello legato alla crescita della scuola nazionale, da lui definita «un tutto organico» (Oliviero, 2007: 204): puntando a un più ampio sviluppo dell'istituzione scolastica, raccolse attorno a sé maestri e professori e, a partire dall'annata 1901-1902, concedette ampio spazio sulla sua rivista anche alle istanze della scuola media e dei suoi insegnanti.

\section{Un caso di cronaca linguistica: Lingua italiana}

A partire dal 1896 fino alla sua cessazione, La Rassegna Scolastica venne pubblicata insieme alla Didattica per le scuole elementari, divenuta dall'ottobre 1902 Didattica pratica per le scuole elementari. Il supplemento ospitava diverse rubriche, il cui fine era quello di offrire un concreto aiuto ai maestri; tra esse talune, come Lingua italiana, Aritmetica e Geografia, comparvero fin dal primo fascicolo $\left(1^{\circ}\right.$ ottobre 1896) e rimasero attive (talvolta con un titolo differente) fino al 1908; altre, invece, ebbero vita effimera ${ }^{6}$.

Lingua italiana ${ }^{7}$, rubrica attenta all'insegnamento e alla corretta trasmissione dell'idioma patrio nelle scuole elementari, cambiò varie volte compilatore. Le sue sorti iniziali furono affidate a Ida Baccini, giornalista, scrittrice e maestra elementare. L'insegnante fiorentina diede alla cronaca linguistica un suo impianto, suddividendola in cinque sezioni (una per ciascuna classe elementare) dotate di ulteriori ripartizioni interne: tra esse, oltre alla Grammatica (presente in tutte le classi ad eccezione della prima), una sottosezione dedicata alla pronunzia e alla corretta riproduzione delle diverse lettere dell'alfabeto (Lettura e scrittura simultanea); una riservata all'allenamento della funzione mnemonica (Esercizio di memoria); una destinata alla dettatura (Lettura e dettatura) e un'ultima dedicata alla composizione (Temi di componimento), nella quale venivano suggerite alcune tracce (con grado di difficoltà diverso a seconda della classe di riferimento) utili per lo svolgimento di brevi elaborati.

L'intento principale della rubrica fu quello di proporre utili consigli per i maestri, corredati da esercizi di varia natura; non si trattava, dunque, di imposizioni o di regole didattiche ben definite, ma di semplici suggerimenti, di ordine linguistico e pedagogico, che i maestri erano liberi di recepire o di rigettare.

Così scriveva la Baccini nel primo numero:

[...] Quindi, o miei cari colleghi, non vi aspettate da me della «didattica» propriamente detta: chè temerei, ciò facendo, di mancare al rispetto che io devo all'esperienza vostra e al vostro savio discernimento. Mi limiterò a darvi dei consigli fraterni, modesti, dei quali sarete padroni di far quel conto che vorrete, o anche di non farne alcuno, se questo vi paresse meglio: mi limiterò a far qualche chiacchierata ai vostri bambini [...] (Baccini, 1896a: 2).

6. Tra queste si ricordino almeno Nozioni varie e Lavori femminili.

7. Da questo momento la rubrica verrà denominata con la sigla LI. 
Per sette numeri, quindi fino al $1^{\circ}$ gennaio 1897, la cronaca fu compilata dalla scrittrice toscana; nell'ottavo fascicolo (16 gennaio 1897) essa risulta mancante e sostituita da una breve comunicazione della Direzione:

Per indisposizione della Sig.ra Ida Baccini mancherà in questo fascicolo il solito articolo sulla lingua italiana. Auguriamo all'egregia nostra collaboratrice una sollecita guarigione, ed intanto pubblichiamo due articoli di interesse didattico, che, ci lusinghiamo, riusciranno graditi ai signori maestri. (Giannitrapani, 1897a: 57)

La lacuna venne compensata con la pubblicazione di due articoli di argomento linguistico ${ }^{8}$. Nel numero successivo, il nono ( $1^{\circ}$ febbraio 1897), la Baccini diede notizia, per mezzo di poche righe indirizzate al Direttore, della cessazione della sua attività collaborativa al supplemento, dovuta al troppo tempo che questa le portava via. II decimo fascicolo (16 febbraio 1897) si aprì con un intervento di Domenico Giannitrapani, volto a tranquillizzare i lettori circa la continuazione della rubrica:

Come ne avevo assunto l'impegno, ho provveduto senza indugio alla continuazione della didattica relativa alla lingua italiana, affidandone l'incarico all'egregio prof. R. Mariani. Egli, con la medesima competenza e amorosa cura della signora Ida Baccini, proseguirà lo svolgimento del programma; e sono convinto che i consigli, i suggerimenti del nuovo Redattore saranno graditi dai signori maestri, ai quali il Mariani è noto per pregevoli lavori didattici 9 . (Giannitrapani, 1897b: 73)

All'interno dello stesso numero il Mariani presentò la sua dichiarazione d'intenti, la quale mostrava notevole affinità con quella proposta dalla Baccini nel primo supplemento:

[...] Al maestro va lasciata piena libertà di scelta sulla forma delle lezioni, sulle applicazioni, sulla estensione degli esercizi, onde adattarli alle esigenze del libro di testo, della qualità e categoria delle scuole e della varietà dei luoghi ov'esse sono. Questi elementi danno carattere speciale alle lezioni sur un medesimo soggetto, pur rimanendo identici il procedimento ed il fine. (Mariani, 1897a: 73)

8. Gli articoli in questione erano: «Dell'insegnamento della lingua nelle nostre scuole elementari» (G. Giansiracusa, 1897: 57-60) e «l compiti scolastici» (G. Curti, 1897: 60-62).

9. Tra i lavori a cui fa riferimento il Giannitrapani vi sono svariate opere inerenti a diverse materie; per la lingua italiana si ricordino Nuovissima grammatica per gli alunni della Quarta e quinta classe elementare a metodo intuitivo corredata di molti esempi, letture, esercizi di lingua e di utili svariate applicazioni (1893); Nuovissimo sillabario, con numerosi esercizi per avviamento al comporre (1893) e Chiari e semplici elementi grammaticali per i primi Corsi delle scuole secondarie (1894); per la matematica, L'aritmetica intuitiva e pratica nelle scuole elementari superiori (1895); Le quattro operazioni in prima classe elementare: Guida pratica e ragionata per l'insegnante (1896); L'aritmetica a metodo ciclico nella seconda classe elementare: Guida pratica con svariati esercizi scritti e di calcolo mentale, secondo i nuovi programmi (1896); dello stesso anno è la medesima Guida pratica per la terza classe; per ciò che riguarda la didattica in generale si menzioneranno: /l dott. Ernesto: consigli per la vita: letture per le scuole complementari e le famiglie (1894) e Ad un'esordiente: Consigli e suggerimenti didattici di un vecchio Maestro (1896). 
Al Mariani si debbono dieci numeri di Lingua italiana (dal 16 febbraio al $1^{\circ}$ luglio 1897); la struttura della rubrica rimase invariata, mantenendo l'originaria suddivisione per classi: unica differenza fu l'inserimento della sottosezione dedicata alla grammatica solo a partire dalla terza classe e non più dalla seconda. Col proposito di approfondire il discorso intorno al Mariani in altra sede, si vedano qui solo alcune sue riflessioni in merito alla lingua e alla sua trasmissione:

[...] Egli [l'insegnante] ricordi, che nella scuola primaria insegnare non vuol dire parlare, ma far parlare; quindi le risposte degli alunni siano anche in dialetto; vengano, poi, subito tradotte in buona lingua dal maestro.

[...] L'allievo deve sempre dire lui; in dialetto, si sa, in gran parte; il maestro aiuta, traduce e fa ripetere; non suggerisce addirittura, per impazienza o cattivo metodo. (Mariani, 1897b: 82);

o ancora:

[...] giornalmente si arricchisca lo scarso patrimonio linguistico dei piccoli alunni di nuovi vocaboli e nuove costruzioni; paziente traduzione delle forme dialettali, che sono il simbolo delle idee che posseggono gli alunni di $1^{\text {a }}$ classe nella sfera della loro esperienza. Siccome ogni provincia ha la sua parlata, che si scosta più o meno dall'italiano in quanto a vocaboli ed a pronunzia, gl'insegnanti devono porre attenzione allo scambio dei suoni, che genera parecchi errori ortografici. (Mariani, 1897c: 89)

Simili considerazioni mostrano uno spiccato interesse per le forme dialettali, ammesse nella prima classe, purché immediatamente corrette dal maestro, il quale non deve precipitarsi a completare le conversazioni degli alunni usando la «buona lingua»; al contrario, deve essere paziente e tradurre, di volta in volta, lemmi ed espressioni dialettali nell'idioma patrio ${ }^{10}$.

Successivamente il testimone passò nelle mani di Leopoldina Zanotti ${ }^{11}$, scrittrice che si occupò della cronaca linguistica dal $1^{\circ}$ ottobre 1897 (il supplemento cessava col chiudersi delle scuole e riprendeva alla loro riapertura), fino al $1^{\circ}$ luglio 1902. A differenza dei suoi predecessori, la Zanotti si mostrò più attenta a discorsi meramente grammaticali, dando a Lingua italiana un volto prettamente normativo: in quest'ottica, propose numerose definizioni delle diverse categorie del discorso, soffermandosi ampiamente sul sistema verbale (con ampie escursioni sull'uso dei modi e sulla dia-

10. Tale atteggiamento è da ricondurre alla diffusione, manifestatasi già in pieno Ottocento, del metodo contrastivo dialetto-italiano. Sulla scorta della Relazione Ascoliana (1875/1982), nonché dell'antecedente Dissertazione del Cesari (1808), tale metodo faceva della dialettofonia il punto di partenza adatto per l'acquisizione dell'italiano. Sull'argomento si vedano Cortelazzo (1983); Gensini (2005) e Demartini (2014: 21-25).

11. Vissuta a cavallo tra Ottocento e Novecento, fu autrice di testi per le scuole elementari; tra le sue opere si ricordano: Il metodo sperimentale nell'intuizione e nell'educazione elementare (1891); Sillabario e prime letture: Metodo fonico (1897); per la collana «Biblioteca scolastica», edita dalla Bemporad, pubblicò Mario e l'Ada: prime letture a compimento del sillabario per le scuole maschili e femminili (1908) e, sempre per la stessa casa editrice, diede alle stampe Verso la luce! Nuovo libro di lettura (1915). Per un approfondimento della figura di Leopoldina Zanotti e dei suoi interventi su Lingua Italiana si rimanda a Monastra (in stampa). 
tesi), ma non tacendo sul genere e sul numero dei nomi, sugli aggettivi, sulle preposizioni e sui diversi complementi.

Talvolta la precisione della definizione andava a cozzare con errori evidenti; a titolo esemplificativo, si potrebbe far cenno ad una spiegazione riguardante i verbi irregolari della seconda coniugazione, comparsa nella sottosezione Grammatica e lingua di una quinta:

Si continui lo studio dei verbi irregolari della seconda coniugazione occupandosi di quelli il cui infinito non finisce in ERE, come fare (facere), dire (dicere) e i loro composti (disfare, rifare, ridire, disdire, contraddire), addurre (aducere), condurre (conducere), porre (ponere), e i suoi composti (apporre, comporre, disporre, frapporre, imporre, interporre, opporre, riporre, sottoporre, sovrapporre, ecc.), trarre (traere). (Zanotti, 1899: 147)

La Zanotti pone in parentesi le forme originarie del verbo latino, dalle quali discendono quelle attuali; tra questi esempi si riscontrano due forme errate: aducere $>$ addurre e traere $>$ trarre. In entrambi i casi le voci latine proposte risultano inesistenti, in quanto i corretti paradigmi sono, rispettivamente, addūco, is, duxi, ductum, ĕre e trăho, is, traxi, tractum, ĕre.

Nel 1902, alla riapertura degli istituti scolastici, la scrittrice, probabilmente per motivi di salute, abbandonò la rubrica, la quale fu immediatamente affidata a Tito Livio Cannone, geniale articolista e direttore delle scuole di Torino del Sangro, che se ne occupò fino al 1908 (anno di cessazione della rivista). Dal 5 ottobre del 1906 la cronaca mutò il proprio titolo in Dalle cose alla lingua; tuttavia non riportò notevoli modifiche in quanto a struttura e intenti, i quali rimasero sostanzialmente inalterati. A tal proposito, sarà forse interessante notare ciò che il Cannone scriveva proprio all'inizio della sua collaborazione con la rivista:

Le cose ci forniscono le sensazioni, le percezioni e quindi le idee; val quanto dire ci danno il materiale per pensare, per parlare, per scrivere. Dunque dalle cose alla lingua: quindi la lettura, la dettatura, la composizione orale e scritta, gli esercizi di memoria, saranno strettamente collegati e coordinati all'insegnamento delle nozioni varie. (Cannone, 1902a: 2)

Dunque, già in questo primo stralcio il Cannone cercava di spiegare quell'espressione che da lì a qualche anno sarebbe poi diventata il nuovo titolo della rubrica.

Particolare attenzione fu da lui rivolta alla correzione degli errori di fonetica attribuibili all'uso del dialetto:

La correzione dei difetti relativi agli organi vocali, che nei bambini non sono ancora completamente sviluppati, e di quelli riferentisi alle forme del parlare dialettale, è cosa di somma importanza. Quindi prima di passare all'insegnamento vero della lettura, il maestro faccia molto parlare i bambini, e con maniere del tutto materne, esiga risposte chiare, espressioni corrette il più ch'è possibile. (Cannone, 1902b: 17); 
si veda inoltre: «[...] Anche i difetti di pronunzia e quelli provenienti dal dialetto locale, vanno presi subito ad osservare, per poterli poi gradatamente correggere.» (Cannone, 1903: 2); si osservi infine il suggerimento presente nel programma di una prima classe: «Conversazioncine dirette a correggere gli errori di pronunzia e quelli derivanti da particolarità fonetiche del dialetto locale.» (Cannone, 1906: 3).

Come si può evincere dalle date, tali consigli venivano dati agli insegnanti sempre ad inizio anno scolastico, come se la correzione di tali difetti fosse un punto di partenza obbligato per la trasmissione della buona lingua, specialmente per le prime classi.

\section{Ida Baccini tra editoria scolastica e pubblicistica periodica}

Vissuta per parecchio tempo all'ombra dei grandi titani della letteratura, Ida Baccini (1850-1911) rivesti un ruolo fondamentale nel panorama della pubblicistica e dell'editoria destinate all'infanzia, nel complesso e intenso passaggio dal XIX e al XX secolo. Dopo il fallimento del suo brevissimo matrimonio (1868-1871), la scrittrice sostenne gli esami per conseguire l'abilitazione all'insegnamento, ottenendo subito l'incarico nel quartiere fiorentino di Rifredi. Mantenutolo fino al 1878, fu costretta a dimettersi forse per via della sua manifesta ostilità nei confronti dell'ordinanza, emanata dall'allora Ministro della pubblica Istruzione Francesco de Sanctis, che introduceva l'insegnamento dell'educazione fisica nelle scuole elementari. Libera da qualsiasi impegno coniugale e scolastico, poté finalmente dedicarsi alla sua passione letteraria: la sua fama fu senz'altro legata a Le memorie di un pulcino, romanzo edito (anonimo) nel 1875 dalla casa editrice Paggi; tuttavia la scrittrice si dedicò alla composizione di numerosi altri testi ${ }^{12}$, alla direzione di due periodici dedicati all'infanzia (Cordelia. Giornale per le giovinette 1884-1911; Giornale dei bambini 1895-1906, poi confluito nel Giornalino della domenica di Vamba) e alla collaborazione con svariate testate giornalistiche (La Vedetta, La Nazione, La Rivista europea, La Gazzetta d'Italia, La Rassegna scolastica, Cenerentola, II Giornale per i bambini, Il Giornale dei fanciulli), alcune delle quali passate del tutto inosservate.

Pur restando sempre estranea al dibattito postunitario sviluppatosi intorno alla lingua italiana, la Baccini espresse in svariate occasioni le sue idee linguistiche, che tanto devono all'amicizia col Dazzi, col Tommaseo e col Vieusseux: scrisse infatti alcuni manuali scolastici, diversi sillabari e numerosi articoli, in cui grande attenzione fu riservata all'insegnamento del nostro idioma. Come già puntualizzato da Elisa De Roberto (De Roberto, 2016), tutto questo materiale andrebbe analizzato dagli storici della lingua, per stabilire quale fosse il modello linguistico delle scuole elementari del tempo. A tal proposito, più che lo spoglio dei manuali scolastici propriamente detti, da sempre poco

\footnotetext{
12. La scrittrice pubblicò svariati testi rispondenti ai suoi interessi preminenti; il mestiere di maestra la legò indissolubilmente al mondo dell'infanzia per il quale scrisse Prime letture composte da una mamma (1877); Seconde letture per le classi elementari (1881); Terze letture per le classi elementari (1882); al mondo degli adulti, invece, si dedicò con la composizione di Vita borghese (1884); Storia di Firenze (1887); Storia di una donna (1888) e Scintille nell'ombra (1910); infine alla figura della donna dedicò Il libro della giovinetta (1886); Come vorrei una fanciulla (1891); Le future mogli (1895) e La fanciulla massaia (1908). Per un elenco approssimativo delle opere bacciniane si rimanda a Marciano (2004: 58-60).
} 
vari nella forma e contenenti definizioni cristallizzate, sarebbe interessante l'analisi delle riflessioni linguistiche della Baccini, espresse soprattutto nei periodici infantili dell'epoca.

\title{
5.1. Le rubriche linguistiche di Ida Baccini
}

Cordelia. Giornale per le giovinette (1881-1942), fondata nel 1881 da Angelo De Gubernatis, rappresenta l'attività giornalistica a cui la scrittrice dedicò gran parte delle forze. Passata sotto la sua direzione nel 1884, acquisì col tempo una fisionomia diversa rispetto alle origini: a mutare fu soprattutto il rapporto con le lettrici, divenuto via via più intimo e confidente. Proprio su questa testata gli interessi linguistici della Baccini trovarono la loro prima regolare manifestazione su rivista: tramite le due rubriche Piccola posta e Concorso di componimento, l'insegnante articolò infatti una serie di considerazioni in materia di didattica linguistica.

Rimandando alla De Roberto per una completa trattazione dell'argomento (De Roberto, 2016), si vedranno in questa sede soltanto alcuni esempi tratti dalla Piccola posta, sezione impiegata dalla Baccini per "correggere" $i$ vizi linguistici presenti nei numerosi elaborati partecipanti al Concorso di componimento.

Per iniziare, si osservi il seguente intervento, nel quale si esorta all'utilizzo di una scrittura chiara, che riecheggi il parlato sorvegliato:

\begin{abstract}
Ciclamino (alunna del Collegio Carletto) - Benedetta la semplicità.... Ė strano: ma in generale gli scolaretti non sanno persuadersi che a scriver come si parla - quando si parla bene, veh? - ci si guadagna un tanto. Crede lei, caro Ciclamino, che certe inversioni, certe frasi come $i$ capelli d'ebano, i pennuti prigionieri, il ruscello che discorre fra i sassi lambendo la sponda ecc. ecc. siano proprio belle? Ormai questi modi hanno fatto il loro tempo ed è meglio lasciarli in pace. Pensieri vogliono essere, carina, e non lusso d'immagini, e non vano sciupio di parole (Baccini, 1887: 102).
\end{abstract}

L'interesse per un uso lineare della scrittura, scevra da qualsiasi sontuosità, è ben ribadita dall'affermazione «non lusso d'immagini, e non vano sciupio di parole», opinione, peraltro, fortemente promossa dal dibattito allora in auge intorno alla necessità di usare parole appropriate per esprimere un determinato concetto ${ }^{13}$.

Sul corretto uso del congiuntivo si veda:

13. Già Ruggiero Bonghi in Lettere critiche (1856) parlava di «lusso vano di locuzioni e di parole». Per la permanenza del dibattito sull'ideale della proprietà espressiva in età postunitaria si rimanda a Polimeni (2011: 81-103). 
Sensitiva: Il babbo, la mamma, le sorelline dormivano: io però ero rimasta alzata a ripassare la storia. Era rimasta alzata? Quel ripassare non è elegante. Riandare col pensiero ad una data cosa non va: ma bensì riandare una data cosa... Mi ricorda (il tic-tac) i più grandi dolori che HO provato. Ella voleva dire che io abbia provato, giacché il che sta a indicare il congiuntivo (Baccini, 1886a: 93);

Esempio nel quale la forma ritenuta errata è addirittura enfatizzata con il maiuscolo; in realtà, come ha evidenziato la De Roberto (De Roberto, 2016), la presenza dell'indicativo in luogo del congiuntivo non rende la relativa affatto scorretta.

Alcuni interventi vedono la Baccini esortare le concorrenti, con tono piuttosto perentorio, alla lettura dei grandi scrittori ottocenteschi e, soprattutto, allo studio delle grammatiche correnti:

A tutte le signorine e signorini che avrebbero bisogno di studiare la grammatica e un po' di buon senso e si ostinano invece a mandarci racconti, versi, e quanto loro passa pel capo, dichiariamo ancora una volta che la Cordelia non tien cattedra né raddrizza le gambe ai cani (Baccini, 1893a: 336).

Per finire, si vedano alcuni esempi relativi all'uso di regionalismi e forme ritenute a vario titolo poco italiane: «Miosotide - Affettuosissimo e gentile. [...] Ma scorretto e spesso sazievole per una certa ostentazione di vernacolo paesano» (Baccini, 1885: 54); o «Maria M. Ancona - Molto grazioso. Lo sciupano alcune voci non schiettamente italiane, come l'indomani, matrina ecc.» (Baccini, 1886b: 93); o ancora «Ciclamino Abito bianco e abito nero - Perché la parola di nuovo scritta dinuovo? [...] E camera da letto non le pare locuzione tutt'altro che italiana? Vi sono forse delle camere senza letto?» (Baccini, 1893b: 354). Forme siffatte sono da evitare a favore di altre definite, dalla stessa, «schiettamente italiane» ${ }^{14}$.

L'interesse per la correzione di forme dialettali può definirsi costante nella scrittrice, se lo si ritrova nelle prime riflessioni linguistiche proposte su Lingua italiana, le cui considerazioni, sicuramente meno incisive, mostrano tuttavia un'apertura maggiore nei confronti del tanto reietto dialetto:

14. Per la spiegazione dei singoli fenomeni si rimanda a De Roberto (2016: 113-114). 
[...] non spaventate il fanciullo con un nuovo vocabolario irto di difficoltà: servitevi, se occorre, del suo stesso dialetto, framettendo di tanto in tanto delle voci italiane su cui vi appoggerete senza ostentazione. Il bambino, non dubitate, noterà quelle voci e le ripeterà compiacendosene. In quale altro modo impariamo noi la lingua materna? Il fanciullo, a tre anni, possiede già un ricco corredo di locuzioni senza aver avuto bisogno per impararle, di precetti o di grammatica. E se le mamme e le balie perdessero il brutto vezzo quando parlano ai loro bambini, di alterar le parole più comuni dell'uso (mommo per bere - pappo per pane - peppo per cappello - pippi per fagiuoli - a cecce per a sedere - to to per percuotere ecc.) i fanciulli verrebbero anche più sollecitamente al possesso della lingua parlata, o del dialetto (Baccini,1896c: 3);

Si veda anche:

L'insegnamento della lingua materna, nella prima classe elementare, non può consistere che in frequenti e ben dirette conversazioni fra il maestro e l'alunno. [...] II maestro, dunque, dovrà tener conto di questa libertà [...] di linguaggio lasciata al fanciullo [...] e si guardi bene durante quei primissimi dialoghi - di mostrare alcun segno di disapprovazione o di disgusto. Il bambino entrerebbe subito in diffidenza e non parlerebbe più (Baccini, 1896b: 2).

Questa sostanziale differenza è da ricondurre al pubblico cui è indirizzata la rubrica: nel caso di Piccola posta, le interlocutrici erano per lo più giovinette che avevano già compiuto il quattordicesimo anno di età ${ }^{15}$; invece, Lingua italiana era espressamente rivolta agli insegnanti delle classi elementari, il cui compito era quello di avvicinare gradualmente i piccoli allievi alla buona lingua, impiegando (limitatamente alle prime classi) conversazioncine tra maestri e discenti: «Le chiacchierate dei ragazzi [...] danno modo all'insegnante di correggere errori di lingua, locuzioni sbagliate, voci dialettali» (Baccini, 1896d: 9); o ancora «Per mezzo della conversazione potrete correggere le pronunzie viziose, gl'intercalari stupidi, i vocaboli impropri o barbari, le offese alla grammatica [...]» (Baccini, 1896l: 41).

Già presente tra le pagine di Cordelia, l'interesse nei confronti di una lingua essenzialmente pratica, che potesse essere impiegata in contesti reali, viene ribadito con forza anche in Lingua italiana:

Oggi, se non vi dispiace, v'insegnerò a scrivere una letterina. [...] La lettera è per chi non c'è, per chi è lontano, per chi non può udire la nostra parola. E siccome la lettera non è altro che la parola scritta, bisogna che essa sia semplice, naturale, senza arzigogoli di frasi, senza sfaccettio di aggettivi inutili, di avverbi pesanti e di punti ammirativi...[...] Molte e molte altre cosette avrei da dirvi intorno a questa specie di componimento che è il più necessario nella vita [...] Non importa affatto che sappiate descrivere un tramonto, indovinare le idee d'un gatto, o interpretare l'amor patrio d'un canarino, ma che impariate a scrivere bene una lettera importa e come! (Baccini, 1896m: 43-44).

15. Addirittura non era rara la presenza di aspiranti scrittori di età superiore, o perfino quella dei genitori stessi. 
Trattasi, in quest'ultima citazione, di un concetto che la Baccini destina ad una quinta classe, costituita da alunni ritenuti evidentemente maturi e, quindi, pronti a usi più complessi del linguaggio.

\subsection{La rubrica Lingua italiana a confronto con le grammatiche coeve}

La prefazione a Nozioni di grammatica italiana (Baccini, 1890) risulta indispensabile per capire a quali modelli grammaticali la Baccini facesse riferimento e, soprattutto, perché:

Resta ora a vedere se tra le Grammatiche pubblicate fin qui ve ne siano molte che [...] si mantengano dentro a quei limiti, variati i quali, l'insegnamento cadrebbe inevitabilmente nell'arido e nel ridicolo. Se nell'esporre i suoi Principii di grammatica il dotto Lambruschini non si perdesse in soverchie lungaggini e non s'indirizzasse più a' maestri che agli scolari, sarebbe difficile rinvenire una Grammatica elementare che rispondesse con maggiore chiarezza ai bisogni intellettuali del fanciullo, in cui fosse osservata con più scrupolosa diligenza la legge di gradazione, dove l'aridità della disciplina venisse compensata e direi quasi nascosta dalla sapiente festività della forma (Baccini, 1890: 4).

Nelle pagine che seguono, tra i modelli considerati positivi, la scrittrice indica Pratica e teorica della lingua italiana (Pera, 1870), dal quale, come si vedrà, mostra di essere fortemente influenzata, non solo per la struttura estremamente dialogica adottata, ma anche per l'impostazione stessa della cronaca: entrambi gli autori, infatti, dopo aver proposto l'argomento, corredano la spiegazione con degli esercizi graduati, nei quali applicare la regola appena esposta.

Al fine di poter stabilire quanto le ideologie linguistiche presenti su Lingua Italiana fossero vicine alle (o lontane dalle) grammatiche allora in uso, verranno, qui di seguito, proposte delle tabelle sinottiche, miranti a raffrontare la rubrica e le grammatiche. Oltre al già citato lavoro di Pera, il confronto prenderà in esame una grammatica del filone purista, nella fattispecie la Grammatichetta della lingua italiana (Moise, 1874) ${ }^{16}$, e una appartenente alla corrente manzoniana, Grammatica italiana (Morandi-Cappuccini, 1894).

Nella Tabella 1 si osservino le diverse modalità impiegate per la spiegazione del verbo, argomento proposto dalla Baccini per la terza classe:

16. Si è preferito optare per questo lavoro, opera compendiata della più vasta Grammatica della lingua italiana (Moise: 1867), in quanto finalizzato direttamente all' uso scolastico. 
Tabella $1^{17}$

\begin{tabular}{|c|c|c|c|}
\hline $\begin{array}{l}\text { LINGUA ITALIANA } \\
\text { BACCINI } \\
(1896)\end{array}$ & $\begin{array}{c}\text { PRATICA E TEORICA } \\
\text { PERA } \\
(1870)\end{array}$ & $\begin{array}{c}\text { GRAMMATICHETTA DELLA } \\
\text { LINGUA ITALIANA } \\
\text { MOISE } \\
(1874)\end{array}$ & $\begin{array}{c}\text { GRAMMATICA ITALIANA } \\
\text { MORANDI-CAPPUCCINI } \\
(1894)\end{array}$ \\
\hline $\begin{array}{l}\text { 1) [...] la parolina è, che, } \\
\text { come avete visto, significa } \\
\text { un'affermazione, si chiama } \\
\text { verbo. E per conseguenza, } \\
\text { tutte le parole che signifi- } \\
\text { cheranno un' affermazione, } \\
\text { saranno verbi. } \\
\text { (Baccini, 1896f: 11) }\end{array}$ & $\begin{array}{l}\text { La parola è nella narrazione } \\
\text { di sopra significa un'affer- } \\
\text { mazione. [...] Le parole che } \\
\text { significano un' affermazio- } \\
\text { ne si chiamano verbi. } \\
\text { (Pera, 1870: } 74 \text { ) }\end{array}$ & $\begin{array}{l}\text { Il Verbo è una parte del di- } \\
\text { scorso, la quale, esprimen- } \\
\text { do tempo, afferma l'azione } \\
\text { o la passione o l'esistenza } \\
\text { o il modo di essere d'una } \\
\text { persona o d'una cosa. } \\
\text { (Moise, 1874: 73) }\end{array}$ & $\begin{array}{l}\text { Il verbo è quella parte del } \\
\text { discorso che esprime un } \\
\text { fatto o un'azione [...] (Mo- } \\
\text { randi e Cappuccini, 1894: } \\
\text { 128) }\end{array}$ \\
\hline $\begin{array}{l}\text { 2) Noi sappiamo dunque } \\
\text { che i numeri dei verbi, } \\
\text { come quelli dei nomi, sono } \\
\text { due: singolare e plurale. Il } \\
\text { verbo è di numero singo- } \\
\text { lare, quando afferma di } \\
\text { una sola persona o di una } \\
\text { sola cosa [...] Il verbo è di } \\
\text { numero plurale, quando af- } \\
\text { ferma di più persone e cose } \\
\text { per es.: I fiori appassiscono, } \\
\text { I soldati combattono, Gli } \\
\text { uccelli volano. (Baccini, } \\
\text { 1896h: 27) }\end{array}$ & $\begin{array}{l}\text { Quanti sono i numeri dei } \\
\text { verbi? I numeri dei verbi, } \\
\text { come quelli dei nomi, sono } \\
\text { due: singolare e plurale. } \\
\text { Quando il verbo è di nume- } \\
\text { ro singolare? Il verbo è di } \\
\text { numero singolare, abbiamo } \\
\text { già detto, quando afferma } \\
\text { di una sola persona, o di } \\
\text { una sola cosa [...]. Quando } \\
\text { il verbo è di numero plura- } \\
\text { le? Un verbo è di numero } \\
\text { plurale quando afferma } \\
\text { di più persone o cose, p. } \\
\text { es. alcuni soldati combat- } \\
\text { tevano, le case rovinarono. } \\
\text { (Pera, 1870: 78) }\end{array}$ & $\begin{array}{l}\text { I Numeri del verbo, come } \\
\text { altrove si è detto, sono } \\
\text { quegli stessi del nome, } \\
\text { cioè il singolare e il plurale, } \\
\text { secondo che il suggetto è } \\
\text { singolare o plurale. (Moise, } \\
\text { 1874: 79) }\end{array}$ & $\begin{array}{l}\text { Il verbo ha due numeri, } \\
\text { come il nome, singolare e } \\
\text { plurale, secondo che il sog- } \\
\text { getto sia singolare o plurale } \\
\text { [... ] (Morandi e Cappuccini, } \\
\text { 1894: 132) }\end{array}$ \\
\hline $\begin{array}{l}\text { 3) «ll maestro non lodava } \\
\text { mai Arturo, quando questi } \\
\text { era presente.» A qual tem- } \\
\text { po appartiene il verbo lo- } \\
\text { dava? Il verbo lodava, oltre } \\
\text { al dare una significazione } \\
\text { di passato, ci dà un'idea di } \\
\text { azione non del tutto com- } \\
\text { piuta, poiché dal maestro } \\
\text { si seguitava a fare. Ebbene: } \\
\text { quel tempo di verbo che } \\
\text { afferma un'azione non del } \\
\text { tutto compiuta, perché si } \\
\text { continua a fare si chiama } \\
\text { imperfetto. Esempio. Io } \\
\text { studiava, [...] (Baccini, } \\
\text { 1896n: 51) }\end{array}$ & $\begin{array}{l}\text { - Socrate filosofo non loda- } \\
\text { va né amico né altra perso- } \\
\text { na quando gli era presente. } \\
\text { - A qual tempo appartiene } \\
\text { il verbo lodava? Oltre alla } \\
\text { significazione di passato, } \\
\text { nella voce lodava si trova } \\
\text { anche l'idea di azione non } \\
\text { al tutto compiuta, perché } \\
\text { da Socrate si continuava a } \\
\text { fare. Come si chiama quel } \\
\text { tempo di verbo che afferma } \\
\text { azione non al tutto com- } \\
\text { piuta, perché si continua a } \\
\text { fare? Quel tempo di verbo } \\
\text { che afferma azione non al } \\
\text { tutto compiuta, perché si } \\
\text { continua a fare, si chiama } \\
\text { tempo imperfetto. [...] } \\
\text { Sing. lo studiava [...] (Pera, } \\
\text { 1870: 81) }\end{array}$ & $\begin{array}{l}\text { Il Passato Imperfetto dinota } \\
\text { che la cosa passata di cui si } \\
\text { parla era incominciata, ma } \\
\text { non finita, nel tempo in cui } \\
\text { si pone avvenuta. - Mentre i } \\
\text { padroni CENAVANO, il ladro } \\
\text { entró in casa. (Moise, 1874: } \\
\text { 77) }\end{array}$ & $\begin{array}{l}\text { L'imperfetto indica che un } \\
\text { fatto, avvenuto nel tempo } \\
\text { passato, ebbe maggiore o } \\
\text { minore durata: Egli ti ascol- } \\
\text { tava. I Romani vestivano la } \\
\text { toga. (1894:131) } \\
\\
\text { L'antica terminazione } \\
\text { va della prima persona } \\
\text { singolare dell'imperfetto } \\
\text { (Io lodava, temeva, crede- } \\
\text { va, vestiva) cede ormai il } \\
\text { posto a vo (lodavo, ecc.) per } \\
\text { evitare la omonimia con la } \\
\text { terza persona. (Morandi e } \\
\text { Cappuccini, 1894: 146) }\end{array}$ \\
\hline
\end{tabular}

17. L/ è posta come primo termine di paragone; le altre grammatiche sono state proposte in ordine cronologico. Per facilitarne la lettura, ogni esempio è stato dotato di numero arabo (solo in $L I$ ). Per questioni di spazio, non sono state riportate tutte le spiegazioni inerenti ai diversi argomenti, ma solo quelle ritenute esemplificative ai fini del nostro lavoro. 
Nei tre esempi proposti si può immediatamente notare un'accentuata somiglianza tra i primi due modelli, molto più discorsivi e dettagliati rispetto agli altri, oltreché esposti con parole pressoché identiche; si noti, a tal proposito, addirittura l'impiego della medesima frase esemplificativa nel caso n.2: i soldati combattono (Baccini) e alcuni soldati combattevano (Pera); o ancora al n.3: Il maestro non lodava mai Arturo quando questi era presente (Baccini) e Socrate filosofo non lodava né amico né altra persona quando gli era presente (Pera). I due autori mostrano, inoltre, di perseguire entrambi il metodo induttivo, permettendo al discente di approdare alla regola tramite l'esempio diretto; le grammatiche del Moise e del Morandi appaiono, invece, legate al modello opposto, il quale antepone l'assioma alle dimostrazioni (es. n.3).

L'esempio n.1 mostra, apparentemente, un'idea di verbo diversa a seconda degli autori: nei primi due casi il verbo è definito un'affermazione, nel Moise esso afferma un'azione e, infine, in Morandi si ritrova una definizione più vicina ai nostri tempi: il verbo serve ad esprimere un'azione o un fatto. In realtà, più che di vera e propria differenza concettuale, si tratta di una versione più sintetica e generalizzata dell'ultima definizione (Morandi).

Riguardo all'uso dell'imperfetto (es. n. 3) si noti che solamente la grammatica del Morandi consiglia di sostituire l'antica terminazione della prima persona singolare -va, con -vo, evitando, in tal modo, confusione tra questa e la terza persona singolare; gli altri modelli, invece, restano saldamente ancorati alla tradizione. Se questo potrebbe passare inosservato per il Pera (data l'altezza cronologica) ed essere considerato del tutto normale per il Moise (data la sua natura espressamente purista), lo stesso non può dirsi per la Baccini, il cui intervento su rivista è addirittura posteriore di due anni rispetto alla grammatica manzoniana ${ }^{18}$. Anche in questo caso, inoltre, la definizione del Morandi è la più sintetica (e priva della notazione sull'incompiutezza dell'azione), mentre quella della Baccini, sulla scorta del Pera, si dilunga per fornire spiegazioni con il minor grado possibile di implicitezza.

18. Già in Petrocchi (1887: 44) si leggeva: «La prima persona dell'imperfetto fa più comunemente Ero e Avevo, che Era e Aveva; e meglio sarà attenersi a quella, perché distingue meglio». 
Nella Tabella 2 si osservi la trattazione dell'avverbio (esposto per la quarta classe):

Tabella $2^{19}$

\begin{tabular}{|c|c|c|c|}
\hline $\begin{array}{l}\text { LINGUA ITALIANA } \\
\text { BACCINI } \\
(1896)\end{array}$ & $\begin{array}{c}\text { PRATICA E TEORICA } \\
\text { PERA } \\
(1870)\end{array}$ & $\begin{array}{c}\text { GRAMMATICHETTA DELLA } \\
\text { LINGUA ITALIANA } \\
\text { MOISE } \\
(1874)\end{array}$ & $\begin{array}{c}\text { GRAMMATICA ITALIANA } \\
\text { MORANDI-CAPPUCCINI } \\
(1894)\end{array}$ \\
\hline $\begin{array}{l}\text { 1) [...] Vi ho già detto che } \\
\text { alcuni di essi accennano al } \\
\text { modo, ossia alla maniera } \\
\text { con cui viene affermata dal } \\
\text { verbo una data azione [...] } \\
\text { questi si chiamano avverbi } \\
\text { di maniera, appunto per- } \\
\text { ché si risolvono colla parola } \\
\text { maniera. Ve ne sono altri } \\
\text { detti di quantità, appunto } \\
\text { perché si risolvono colla } \\
\text { parola quantità[...] Vi sono } \\
\text { avverbi detti di luogo, che si } \\
\text { possono risolvere colla pa- } \\
\text { rola luogo, o anche rispon- } \\
\text { dendo alla parola dove [...] } \\
\text { Gli avverbi di tempo sono } \\
\text { quelli che si possono risol- } \\
\text { vere colla parola tempo, } \\
\text { o anche rispondendo alla } \\
\text { parola quando [...] Vi sono } \\
\text { poi avverbi d'ordine [.... Ve } \\
\text { ne ha di preferenza [...] Vi } \\
\text { sono avverbi di similitudine, } \\
\text { i quali si usano quando } \\
\text { si vogliono confrontare } \\
\text { due cose[...] Gli avverbi } \\
\text { di dubbio, sono quelli che } \\
\text { significano probabilità o } \\
\text { incertezza [...] (Baccini, } \\
\text { 1896i: 36) }\end{array}$ & $\begin{array}{l}\text { [...] Vi sono dunque varie } \\
\text { specie di avverbi? Sì, vi } \\
\text { sono molte specie di avver- } \\
\text { bi, secondo il loro diverso } \\
\text { significato, cioè avverbi di } \\
\text { maniera, di quantità, di luo- } \\
\text { go, di tempo, di preferenza, } \\
\text { di similitudine, di dubbio. } \\
\text { (Pera, 1870: 136-137) }\end{array}$ & $\begin{array}{l}\text { Per rispetto alla diversa } \\
\text { loro significazione si divido- } \\
\text { no gli avverbj in Avverbj di } \\
\text { tempo, di luogo, di quantità, } \\
\text { di similitudine, di qualità o } \\
\text { di modo, di affermazione, di } \\
\text { negazione. } \\
\text { (Moise, 1874: 120)1 }\end{array}$ & $\begin{array}{l}\text { Il maggior numero degli } \\
\text { avverbi si compone de' } \\
\text { qualificativi, che per lo } \\
\text { più si riferiscono al verbo, } \\
\text { qualificandone l'azione; e } \\
\text { hanno perciò, rispetto a lui, } \\
\text { lo stesso ufficio che hanno, } \\
\text { rispetto al nome, gli ag- } \\
\text { gettivi qualificativi: [...] Lo } \\
\text { guardò amorevolmente (Gli } \\
\text { diede uno sguardo amore- } \\
\text { vole) [...] (1894: 205) } \\
\text { Gli altri avverbi, si suol } \\
\text { dividerli in molte classi, } \\
\text { secondo le diverse relazioni } \\
\text { che esprimono; e le relazio- } \\
\text { ni principali sono: } \\
\text { a) La maniera: come, } \\
\text { comunque, cosi [...] b) Il } \\
\text { tempo [...] c) Il luogo [...] d) } \\
\text { La quantità [...] e) L'ordine } \\
\text { [...] f) L'affermazione [...] } \\
\text { g) La negazione [...] h) Il } \\
\text { dubbio [...] i) L'aggiunzione } \\
\text { [...] (Morandi e Cappuccini, } \\
\text { 1894: 206-207) }\end{array}$ \\
\hline
\end{tabular}

L'avverbio, categoria dei mal definiti per antonomasia, presenta qui delle sostanziali divergenze riguardanti la classificazione delle diverse tipologie: in LI e in Teorica e pratica mancano le categorie avverbiali di affermazione e negazione, presenti invece sia nel modello purista, che in quello manzoniano (dove figurano anche gli avverbi d'aggiunzione). Forse sarebbe più interessante osservare che gli avverbi definiti di maniera o modo nei primi due modelli diventano di qualità in Moise e qualificativi in Morandi-Cappuccini, dove, addirittura, sotto l'etichettatura di avverbi di maniera, si catalogano le forme come, comunque e cosi. Nulla di nuovo, dunque, per tale categoria, soggetta da sempre alla scarsa coerenza dei manuali e dei loro compilatori.

19. Le considerazioni rinvenute sulla Grammatichetta della lingua italiana del Moise e sulla Grammatica italiana di Morandi-Cappuccini non riportano alcuna definizione, ma esclusivamente una serie di esempi (non trascritti in tabella) per ogni categoria avverbiale menzionata. 
Per quanto concerne invece il nome (trattato nella seconda classe), la differenza più rilevante tra i diversi modelli consiste nella suddivisione dei sostantivi in quattro declinazioni, proposta ancora sia da Pera (Pera, 1870: 33-37), sia da Morandi (Morandi-Cappuccini, 1894: 54-65)20; ripartizione che invece risulta totalmente assente in $\mathrm{L}$, dove la Baccini si concentra unicamente su questioni di numero e di genere: «Ogni persona e ogni cosa ha il suo nome [...] I nomi servono ad indicare le persone e le cose» (Baccini, 1896e: 10); "Quando, dunque, un nome indica una cosa sola o una sola persona, si dice che quel nome appartiene al numero singolare. Ma se il nome indicasse più cose o più persone, apparterrebbe al numero plurale. Vi pare d'aver capito? [...] II nome penna appartiene al singolare, ma penne al plurale» (Baccini, 1896g: 26).

\section{Conclusioni}

Abbiamo avuto modo di vedere come gli interventi bacciniani abbiano fatto di Lingua italiana una cronaca linguistica sostanzialmente bipolare, ospitante al suo interno riflessioni di ordine grammaticale, miste ad altre di natura ideologico-linguistica. Il volto della rubrica, come già accennato, non fu però sempre il medesimo: se, infatti, l'originaria struttura pentapartita fu mantenuta inalterata da tutti i collaboratori, diversa sorte toccò invece ai contenuti (ideologico-linguistici con Mariani e Cannone, grammaticali con la Zanotti).

Rubrica ben articolata e completa, trattante i diversi ambiti del nostro idioma (dalla fonetica ai temi di componimento), Lingua italiana si proponeva come un utile strumento da affiancare (non da sostituire) ai manuali scolastici d'uso. A tal proposito, facendo riferimento a ciò che è stato osservato nelle pagine precedenti, si potrebbe, forse, tentare di abbozzare il profilo ideologico-linguistico della Baccini.

Studi intorno alla sua produzione extrascolastica (De Roberto, 2016) ci permettono di rilevare una sostanziale propensione per scelte linguistiche di ordine manzoniano, che tuttavia, di tanto in tanto, cedono il passo a qualche sporadico tratto premanzoniano. In merito a ciò si osservi il persistere di forme verbali con enclisi pronominale (ad esempio avvi ed èvvi): siffatte scelte ribadiscono, ancora una volta, la forte influenza subita dalla grammatica di Francesco Pera (1870), nella quale si legge:

20. Il Moise non parla invece di declinazioni, ma tra gli «accidenti del nome» menziona (oltre al Genere, al Numero e all'Alterazione) il Rapporto che «corrisponde al Caso de' Latini» e «ci dimostra i varj officj ch'esso [il nome] esercita nel discorso» (1874: 47). 
Le particelle pronominali si collocano o prima o dopo certe parole [verbi], p. es. si nutre e nutresi. [...] Le particelle pronominali che stanno prima sono separate, p. es. ti perdonò; quelle che stanno dopo sono congiunte, p. es. perdonòtti. [...] In questo esempio si è raddoppiata la consonante della particella ti, perché quando tali particelle sono congiunte a parole che terminano con l'accento, raddoppiano la consonante [...] (Pera, 1870: 66).

Esse mostrano, nel contempo, un netto distacco dalla normativa proposta nella grammatica di Morandi-Cappuccini (1894), contemporanea all'autrice: «Mi, ti, lo, la, ci, vi, li, le, ne, comunemente, nella prosa, si premettono al verbo; ma lo seguono quando esso sia imperativo, o infinito, o gerundio, o participio.» (Morandi-Cappuccini, 1894: 117).

Oscillazioni simili sono state riscontrate anche nei suoi interventi normativi su Lingua Italiana: se per quanto concerne il nome, infatti, l'autrice si propone come innovatrice, abbandonando il tradizionale sistema dei casi (ancora proposto da Morandi-Cappuccini), lo stesso non può essere affermato per il verbo, sezione in cui la Baccini, suggerendo l'impiego dell'antica desinenza dell'imperfetto, mostra di essere ancora saldamente legata alla tradizione.

Non va taciuta, inoltre, la sua spiccata modernità nei confronti delle questioni allora più dibattute; modernità mostrata sia nel favorire l'impiego di forme dialettali nelle conversazioni con gli alunni (sia pure limitatamente alla prima classe), sia anche nel proporre (o meglio, imporre) l'uso di una scrittura chiara e appropriata che non facesse sfoggio di alcuna sontuosità, né di virtuosismi stilistici o poetismi d'accatto, nonostante l'invito, talora, a preferire forme lessicali più ricercate alla più comuni.

Anche gli interventi su rivista, dunque, conducono alla medesima conclusione: complessità e interesse della figura di Ida Baccini, scrittrice, insegnante e giornalista il cui profilo ideologico-linguistico risulta di carattere preminentemente filomanzoniano, inframmezzato, talora, da rari tratti filotradizionalisti. 


\section{Bibliografia}

Ascoli, Graziadio Isaia (1875/1982), «Relazione al IX Congresso Pedagogico Italiano», in Marino Raicich (1981), Scuola, cultura e politica da De Sanctis a Gentile, Pisa, Nistri-Lischi, p. 425-431.

Baccini, Ida (1885), [Senza titolo], nella rubrica Piccola posta, Cordelia. Giornale per le giovinette, $n^{\circ} 5$, p. 54.

Baccini, Ida (1886a), [Senza titolo], nella rubrica Piccola posta, Cordelia. Giornale per le giovinette, $n^{\circ} 5$, p. 93.

Baccini, Ida (1886b), [Senza titolo], nella rubrica Piccola posta, Cordelia. Giornale per le giovinette, n 5, p. 93.

Baccini, Ida (1887), [Senza titolo], nella rubrica Piccola posta, Cordelia. Giornale per le giovinette, $n^{\circ} 7$, p. 102.

Baccini, Ida (1890), Nozioni di grammatica italiana esposte secondo il metodo intuitivo ad uso delle scuole elementari, Firenze, Bemporad e Figlio, coll. «Biblioteca scolastica» [1 $1^{\mathrm{a}}$ ed. 1882].

Baccini, Ida (1893a), [Senza titolo], nella rubrica Piccola posta, Cordelia. Giornale per le giovinette, $n^{\circ} 14$, p. 336.

Baccini, Ida (1893b), [Senza titolo], nella rubrica Piccola posta, Cordelia. Giornale per le giovinette, $n^{\circ} 14$, p. 354.

Baccini, Ida (1896a), [Senza titolo], nella rubrica Lingua italiana, Didattica per le scuole elementari, supplemento della rivista La Rassegna Scolastica, $n^{\circ}$ 1, p. 2.

Baccini, Ida (1896b), [Senza titolo], nella rubrica Lingua italiana, Didattica per le scuole elementari, supplemento della rivista La Rassegna Scolastica, $\mathrm{n}^{\circ}$ 1, p. 2.

Baccini, Ida (1896c), [Senza titolo], nella rubrica Lingua italiana, Didattica per le scuole elementari, supplemento della rivista La Rassegna scolastica, $n^{\circ} 1$, p. 3.

Baccini, Ida (1896d), [Senza titolo], nella rubrica Lingua italiana, Didattica per le scuole elementari, supplemento della rivista La Rassegna Scolastica, nº 1, p. 9.

Baccini, Ida (1896e), [Senza titolo], nella rubrica Lingua italiana, Didattica per le scuole elementari, supplemento della rivista La Rassegna Scolastica, nº 1, p. 10.

Baccini, Ida (1896f), [Senza titolo], nella rubrica Lingua italiana, Didattica per le scuole elementari, supplemento della rivista La Rassegna Scolastica, n 1 , p. 11.

Baccini, Ida (1896g), [Senza titolo], nella rubrica Lingua italiana, Didattica per le scuole elementari, supplemento della rivista La Rassegna Scolastica, nº 1, p. 26.

Baccini, Ida (1896h), [Senza titolo], nella rubrica Lingua italiana, Didattica per le scuole elementari, supplemento della rivista La Rassegna Scolastica, nº 1, p. 27. 
Baccini, Ida (1896i), [Senza titolo], nella rubrica Lingua italiana, Didattica per le scuole elementari, supplemento della rivista La Rassegna Scolastica, nº 1, p. 36.

Baccini, Ida (1896l), [Senza titolo], nella rubrica Lingua italiana, Didattica per le scuole elementari, supplemento della rivista La Rassegna Scolastica, nº 1, p. 41.

Baccini, Ida (1896m), [Senza titolo], nella rubrica Lingua italiana, Didattica per le scuole elementari, supplemento della rivista La Rassegna Scolastica, n 1 , p. 43-44.

Baccini, Ida (1896n), [Senza titolo], nella rubrica Lingua italiana, Didattica per le scuole elementari, supplemento della rivista La Rassegna Scolastica, n 1, p. 51.

Baccini, Ida (2004), La mia vita: ricordi autobiografici, a cura di Lorenzo Cantatore, Milano, Unicopli.

Barbagli, Marzio (1974), Disoccupazione intellettuale e sistema scolastico in Italia, Bologna, Il Mulino.

Bonetta, Gaetano (1981), Istruzione e società nella Sicilia dell'Ottocento, Palermo, Sellerio.

Bonetta, Gaetano (1990), «Scuola laica e scuola cattolica in Sicilia tra '800 e '900» in Chiesa e società urbana in Sicilia (1890-1920), Catania, Atti del convegno di Studi, p. 213-42.

Bonghi, Ruggiero (1856), Lettere critiche di Ruggiero Bonghi: perché la letteratura italiana non sia popolare in Italia, Milano, Colombo-Perelli.

Cannone, Tito Livio (1902a), [Senza titolo], nella rubrica Lingua italiana, Didattica pratica per le scuole elementari, supplemento della rivista La Rassegna Scolastica, nº 7, p. 2.

Cannone, Tito Livio (1902b), [Senza titolo], nella rubrica Lingua italiana, Didattica pratica per le scuole elementari, supplemento della rivista La Rassegna Scolastica, nº 7, p. 17.

Cannone, Tito Livio (1903), [Senza titolo], nella rubrica Lingua italiana, Didattica pratica per le scuole elementari, supplemento della rivista La Rassegna Scolastica, nº 8, p. 2.

Cannone, Tito Livio (1906), [Senza titolo], nella rubrica Dalle cose alla lingua, Didattica pratica per le scuole elementari, supplemento della rivista La Rassegna Scolastica, nº 11, p. 3.

Catricalà, Maria (1991), Le grammatiche scolastiche dell'italiano edite dal 1860 al 1918, Firenze, Accademia della Crusca.

Cesari, Antonio (1810/2002), Dissertazione sopra lo stato presente della lingua italiana, Roma-Padova, Antenore.

Chiosso, Giorgio (1992), I periodici scolastici nell'Italia del secondo Ottocento, Brescia, Editrice La Scuola.

Cortelazzo, Manlio (1983), «Dall'abate Cesari a Tullio De Mauro. Il dialetto nei libri per le scuole venete», in Manlio Cortelazzo (ed.), Guida ai dialetti veneti, Padova, CLEUP, p. 85-122.

Curti, G., (1897), «I compiti scolastici», Didattica per le scuole elementari, supplemento della rivista La Rassegna Scolastica, n¹, p. 60-62. 
Demartini, Silvia (2014), Grammatica e grammatiche in Italia nella prima metà del Novecento, Firenze, Franco Cesati Editore.

De Roberto, Elisa (2016), «"A scriver come si parla si guadagna un tanto”. Ida Baccini e l'insegnamento dell'italiano», in Giuseppe Polimeni e Franco Pierno (ed.), Nuovo italiano, Firenze, Cesati, p. 91115.

Gensini, Stefano (2005), Breve storia dell'educazione linguistica dall'Unità a oggi, Roma, Carocci.

Giannitrapani, Domenico (1897a), [Senza titolo], nella rubrica Lingua italiana, Didattica per le scuole elementari, supplemento della rivista La Rassegna Scolastica, nº 1, p. 57.

Giannitrapani, Domenico (1897b), [Senza titolo], nella rubrica Lingua italiana, Didattica per le scuole elementari, supplemento della rivista La Rassegna Scolastica, nº 1, p. 73.

Giannitrapani, Domenico (1899), «Per intenderci», La Rassegna Scolastica, n 4, p. 478.

Giansiracusa, G[aetano] (1897), «Dell'insegnamento della lingua nelle nostre scuole elementari», Didattica per le scuole elementari, supplemento della rivista La Rassegna Scolastica, nº 1, p. 57-60.

Liberto, M.(1880-1881), «La stampa scolastica italiana», La scuola italiana, nº 1, p. 86-87.

Marchetti Chini, Bice (1891), Ida Baccini, Firenze, Le Monnier.

Marciano, Annunziata (2004), Alfabeto ed educazione. I libri di testo nell'talia post-risorgimentale, Milano, Franco Angeli, p. 58-60.

Mariani, Raffaele (1897a), [Senza titolo], nella rubrica Lingua italiana, Didattica per le scuole elementari, supplemento della rivista La Rassegna Scolastica, nº 1, p. 73.

Mariani, Raffaele (1897b), [Senza titolo], nella rubrica Lingua italiana, Didattica per le scuole elementari, supplemento della rivista La Rassegna Scolastica, nº 1, p. 82.

Mariani, Raffaele (1897c), [Senza titolo], nella rubrica Lingua italiana, Didattica per le scuole elementari, supplemento della rivista La Rassegna Scolastica, nº 1, p. 89.

Moise, Giovanni (1874), Grammatichetta della lingua italiana, Firenze, Tip. Del Vocabolario.

Monastra, Alessandra (in stampa), «Contributi alla storia della didattica dell'italiano: gli interventi di Leopoldina Zanotti in Lingua italiana», La Lingua italiana. Storia, strutture, testi, Pisa/Roma, Fabrizio Serra editore.

Morandi, Luigi e Cappuccini, Giulio (1894), Grammatica italiana. Regole ed esercizi per uso delle scuole ginnasiali, tecniche e normali, Torino, Paravia.

Oliviero, Stefano (2007), «Le riviste per la scuola. Periodici Bemporad dal 1890 al fascismo» in Carla Ida Salviati, Aldo Cecconi (ed.), Paggi e Bemporad, editori per la scuola. Libri per leggere, scrivere e far di conto, Firenze, Giunti, p. 193-227. 
Ottino, Giuseppe (1875), La stampa periodica, il commercio dei libri e la tipografia in Italia, Milano, Brigola.

Parato, Giovanni (1867), «Ai nostri Associati», La guida del maestro elementare italiano, n 3, p. 801 802.

Pera, Francesco (1870), Pratica e teorica della lingua italiana, Firenze, Felice Paggi editore. [1ª ed. 1863].

Petrocchi, Policarpo (1887), Grammatica della lingua italiana: per le scuole elementari inferiori, Milano, Treves.

Polimeni, Giuseppe (2011), «ll troppo e il vano della lingua: l'ideale della proprietà espressiva dal dibattito linguistico alla scuola italiana dopo l'Unità», La lingua italiana. Storia, strutture, testi, $\mathrm{n}^{\circ} \mathrm{7}$, p. 81-103.

Porciani, Ilaria (ed.) (1983), Editori a Firenze nel secondo Ottocento, Firenze, Olschki.

Zanotti, Leopoldina (1899), [Senza titolo], nella rubrica Lingua italiana, Didattica per le scuole elementari, supplemento della rivista La Rassegna Scolastica, n³ 3, p. 147. 